\title{
Some analysis on a fractional differential equation with a right-hand side which has a discontinuity at zero
}

\author{
Müfit Şan ${ }^{1}$ (D), Uğur Sert*2 (D) \\ ${ }^{1}$ Department of Mathematics, Faculty of Science, Çankırı Karatekin University, Tr-18100, Cankırı, \\ Turkey \\ ${ }^{2}$ Department of Mathematics, Faculty of Science, Hacettepe University, Beytepe, Tr-06800, Ankara, \\ Turkey
}

\begin{abstract}
In this article, we consider an initial value problem for a nonlinear differential equation with Riemann-Liouville fractional derivative. By proposing a new approach, we prove local existence and uniqueness of the solution when the nonlinear function on the right hand side of the equation under consideration is continuous on $(0, T] \times \mathbb{R}$.
\end{abstract}

Mathematics Subject Classification (2010). 34A08, 37C25, 34A12, 74G20, 26A33

Keywords. fractional differential equations, mean value theorem, Nagumo-type uniqueness, Peano-type existence theorem

\section{Introduction and motivation}

The birth of fractional calculus dates back to the early days of differential calculus. The number of researchers working on fractional calculus were inadequate when compared to researchers in differential calculus studies, eventually there has been no progress on this field in a reasonable amount of time. However, the interest in fractional calculus and fractional differential equations has increased considerably for the last three decades. This has led to a rapidly development in fractional calculus by virtue of the techniques, methods and results used in the ordinary differential calculus. Evidently, a substantial part of the interest in this subject derives from initial-value problems (IVPs) and boundary-value problems (BVPs) for the fractional differential equations with fractional derivatives such as Riemann-Liouville (RL), Caputo, Caputo-Fabrizio, Grünwald-Letkinov etc. Existence and uniqueness of solution for the IVPs and BVPs were studied by many researchers (see for example $[2,5,7,11,13-16,21-26])$. These articles deal with the qualitative properties of solutions for the equations with continuous right-hand side. However, in this paper, we address an equation with Riemann-Liouville derivative

$$
D^{a} u(x)=f(x, u(x)),
$$

such that $f(x, t)$ has a discontinuity at $x=0$ where $(x, t) \in[0, T] \times \mathbb{R}$.

\footnotetext{
*Corresponding Author.

Email addresses: mufitsan@karatekin.edu.tr (M. Şan), usert@hacettepe.edu.tr (U. Sert)

Received: 14.01.2019; Accepted: 18.12.2019
} 
Throughout the text, by an equation with continuous right-hand side, we mean a function $f(x, t)$ which is continuous on $[0, T] \times \mathbb{R}$, and a discontinuous right-hand side will refer a function $f(x, t)$ which has a discontinuity at $x=0$.

Considering the coincidence of first order RL derivative with ordinary derivative (see $[20,21]$ ), real-world applications of an equation with RL derivative which has discontinuous right-hand side would be enlightening from many problems arising from mechanics, electrical engineering and the theory of automatic control. Differential equations with discontinuous right-hand sides, in particular with a function $f(x, t)$ which is discontinuous in $x$ and continuous in $t$ were studied widely in the literature. For these studies, we refer the book of Filippov [12] (also the references cited therein), which is accepted as a basic source for the general theory of discontinuous dynamical systems.

In this paper, we investigate the following initial value problem for a differential equation with RL fractional derivative:

$$
\left\{\begin{array}{l}
D^{a} u(x)=f(x, u(x)), \quad x>0 \\
u(0)=u_{0},
\end{array}\right.
$$

where $0<a<1, u_{0} \neq 0$ is a real number and the function $f$ will be specified later. The operator, $D^{a}$ represents RL fractional derivative of order $a$, which is defined by combining the ordinary derivative and RL fractional integral $I^{a}$ as follows:

$$
D^{a} u(x):=\frac{d}{d x}\left[{ }_{x} I^{1-a} u\right] \text { with } I^{a} u(x):=\frac{1}{\Gamma(a)} \int_{0}^{x} \frac{u(\xi)}{(x-\xi)^{1-a}} d \xi,
$$

where, $\Gamma(\cdot)$ is the well-known Gamma function.

Problem (1.1) with continuous right-hand side was first discussed in [16] and it was claimed that the continuous solution of the problem exists on the interval $[0, T]$. Nevertheless, Zhang [26] gave an example which indicates that the initial condition $u(0)=u_{0}$ (except $u_{0}=0$ ) is not suitable for studying the existence of continuous solution of (1.1), when the function $f$ is continuous on $[0, T] \times \mathbb{R}$. Accordingly, Şan [23] considered this problem with $f$ which satisfies the following conditions:

(A.1) $f(x, t)$ and $x^{a} f(x, t)$ are continuous on $(0, T] \times \mathbb{R}$ and $[0, T] \times \mathbb{R}$ respectively, (A.2) $\left.x^{a} f\left(x, u_{0}\right)\right|_{x=0}=\frac{u_{0}}{\Gamma(1-a)}$.

In [23], it is proved that the condition (A.2) is necessary for the existence of the continuous solution of problem (1.1). The author also gave a partial answer to the question of the existence of continuous solutions to (1.1). Problem (1.1) represents a system, accordingly the initial condition must be independent of the tools we analyze. Based on this view, after a discussion with Manuel D. Ortigueira (see also $[18,19]$ ) we draw a conclusion that it would be more accurate to discuss the nonexistence of a continuous solution of (1.1) instead of querying the suitability of the initial datum. In fact, if there were a continuous solution $u$ of $(1.1)$ when $f$ is continuous on $[0, T] \times \mathbb{R}$, then by using the compositional relation $u(x)=I^{a} D^{a} u(x)$ proved in Proposition 2.4 in [5] and $u \in C[0, T]$, $D^{a} u \in C[0, T]$, it would be shown that

$$
u(x)=\frac{1}{\Gamma(a)} \int_{0}^{x} \frac{f(\xi, u(\xi))}{(x-\xi)^{1-a}} d \xi, \quad x \in[0, T] .
$$

Thus, by the continuity of $f(x, u(x))$ on $[0, T]$ we obtain,

$$
0 \neq u_{0}=\lim _{x \rightarrow 0^{+}} u(x)=\frac{1}{\Gamma(a)} \int_{0}^{1} \frac{\lim _{x \rightarrow 0^{+}} x^{a} f(x t, u(x t))}{(1-t)^{1-a}} d t=0,
$$

which yields a contradiction. This implies that problem (1.1) with continuous right-hand side and initial condition $u(0)=u_{0} \neq 0$ does not have a continuous solution $u(x)$ on the closed interval. 
In the following section, under the conditions (A.1)-(A.2), we first prove the existence of continuous solutions of (1.1) by using Leray-Schauder alternative. However, as seen in the sequel, this theorem does not inform us about the existence interval of the solution. In the literature, there are some researchers (e.g. [3,10] and references therein) who worked on the existence interval and maximal existence interval of the solution for certain fractional differential equations. For example, Mustafa and Baleanu [3] presented a method by virtue of Leray-Schauder alternative to gain a better estimate for the existence interval of the continuous solutions to the problem they considered. Such an approach cannot be applied directly to the analysis of (1.1) hence, developing a new technique and using Schauder's fixed point theorem, we prove a Peano-type existence theorem for (1.1) which explicitly shows the existence interval of the solution.

For the uniqueness of problems similar to (1.1), there exist some Nagumo-type uniqueness results (see, for example, $[7,11,15,22]$ ) which were proved by the technique and approach developed by Diaz [6]. Apart from the Nagumo-type conditions, for the functions satisfying certain conditions, Diethelm [7] and Odibat [17] employed fractional mean value theorems to establish the uniqueness. We verify the existence and uniqueness of the continuous solution of (1.1) when the function $f$ fulfills a Nagumo-type condition. Unlike the techniques used in the book [1], in this paper, we present a novel technique to prove the uniqueness of (1.1) by combining with a fractional mean value theorem for functions $u \in C[0, T]$ satisfying the inclusions, $D^{a} u \in C(0, T]$ and $x^{a} D^{a} u \in C[0, T]$.

\section{Preliminaries and main results}

Before proceeding to study problem (1.1), we remind some basic facts from functional analysis. At first, we give a lemma which shows the equivalence of the solutions of problem under consideration and solutions of the corresponding integral equation (1.2) (see, [23, 24]).

Lemma 2.1. Under the conditions of (A.1)-(A.2), $u \in C[0, T]$ is a solution of problem (1.1) if and only if $u \in C[0, T]$ is a solution of (1.2).

Let us define the operator $\mathcal{M}: C[0, T] \mapsto C[0, T]$ associated with integral equation (1.2) as follows:

$$
\mathcal{M} u(x):=\frac{1}{\Gamma(a)} \int_{0}^{x} \frac{f(\xi, u(\xi))}{(x-\xi)^{1-a}} d \xi, \quad x \in[0, T] .
$$

Since, the fixed points of the operator $\mathcal{M}$ coincide with the solutions of integral equation (1.2), our goal is to find out the fixed points of operator $\mathcal{M}$ by applying following theorems $[4,8,9]$.

Theorem 2.2 (Schauder's fixed-point theorem). Let $X$ be a real Banach space, $B \subset X$ nonempty closed bounded and convex, $\mathcal{M}: B \rightarrow B$ compact. Then, $\mathcal{M}$ has a fixed point.

Remark 2.3. In applications, it is usually too difficult or impossible to establish a set $B$ so that $\mathcal{M}(B) \subseteq B$ (see, [8]). Therefore, it will be convenient to consider operator $\mathcal{M}$ that maps the whole space $X$ into $X$ to overcome this difficulty. The following result is intimately associated with what we stated above.

Theorem 2.4 (Leray-Schauder alternative). Let $X$ be normed linear space and $\mathcal{M}: X \rightarrow$ $X$ be a completely continuous (compact) operator. Then, either there exists $u \in X$ such that

$$
u=\mathcal{M} u
$$

or the set

is unbounded.

$$
\mathcal{E}(\mathcal{M}):=\{u \in X: u=\mu \mathcal{M}(u) \text { for a certain } \mu \in(0,1)\}
$$


The compactness of operator $\mathcal{M}$ was proved previously by Theorem 2.5 in [23], therefore it is sufficient to show that remaining conditions of the fixed point theorems given above will be fulfilled. The first existence theorem for problem (1.1) is as follows:

Theorem 2.5. Let conditions (A.1)-(A.2) be satisfied for $0<T<\infty$. Moreover, assume that there exists a positive real number $M$ such that

$$
M=\sup _{(x, t) \in[0, T] \times \mathbb{R}}\left|x^{a} f(x, t)-\frac{u_{0}}{\Gamma(1-a)}\right| .
$$

Then, problem (1.1) admits at least one continuous solution $u \in C([0, T])$.

Proof. We employ Leray-Schauder alternative and it is sufficient to show that the set $\mathcal{E}(\mathcal{M})$ defined in $(2.2)$ is bounded. For an arbitrary $u \in \mathcal{E}(\mathcal{M})$ one has

$$
\begin{aligned}
|u(x)| & \leq \mu \frac{1}{\Gamma(a)}\left|\int_{0}^{x} \frac{f(\xi, u(\xi))}{(x-\xi)^{1-a}} d \xi\right| \\
& <\frac{1}{\Gamma(a)}\left|\int_{0}^{x} \frac{f(\xi, u(\xi))-\xi^{-a} \frac{u_{0}}{\Gamma(1-a)}+\xi^{-a} \frac{u_{0}}{\Gamma(1-a)}}{(x-\xi)^{1-a}}\right| \\
& \leq M \Gamma(1-a)+\frac{\left|u_{0}\right|}{\Gamma(1-a) \Gamma(a)} \int_{0}^{x} \frac{1}{\xi^{a}(x-\xi)^{1-a}} d \xi \\
& =M \Gamma(1-a)+\left|u_{0}\right| .
\end{aligned}
$$

Thus, for any $u \in \mathcal{E}(\mathcal{M})$ we get

$$
\sup _{x \in[0, T]}|u(x)|<M \Gamma(1-a)+\left|u_{0}\right|,
$$

which yields that $\mathcal{E}(\mathcal{M})$ is bounded. As a result of Leray-Scauder alternative, (1.1) admits at least one solution in $C[0, T]$.

We now, give a mean value theorem for RL derivative to establish existence and uniqueness results for problem (1.1). For the proof of this theorem, we follow the path outlined in [7] and [17].

Lemma 2.6. Let $u \in C[0, T]$ with $D^{a} u \in C(0, T]$ and $x^{a} D^{a} u \in C[0, T]$ for $0<a<1$. Then, there exists a function $\lambda(x), \lambda:[0, T] \rightarrow(0, T)$ with $0<\lambda(x)<x$ such that

$$
u(x)=\Gamma(1-a)(\lambda(x))^{a} D^{a} u(\lambda(x))
$$

is fulfilled for all $x \in[0, T]$.

Proof. By using the fact $u(x)=I^{a} D^{a} u(x)$ and by mean value theorem of integral calculus we have,

$$
\begin{aligned}
u(x) & =\frac{1}{\Gamma(a)} \int_{0}^{x} \frac{D^{a} u(\xi)}{(x-\xi)^{1-a}} d \xi \\
& =\frac{1}{\Gamma(a)} \int_{0}^{x} \frac{\xi^{a} D^{a} u(\xi)}{\xi^{a}(x-\xi)^{1-a}} d \xi \\
& =\frac{(\lambda(x))^{a} D^{a} u(\lambda(x))}{\Gamma(a)} \int_{0}^{x} \frac{1}{\xi^{a}(x-\xi)^{1-a}} d \xi \\
& =\Gamma(1-a)(\lambda(x))^{a} D^{a} u(\lambda(x)),
\end{aligned}
$$

where $\lambda=\lambda(x) \in(0, x)$ for all $x \in[0, T]$. 
Remark 2.7. In [17], the dependence of $\lambda$ on $x$ was not clearly expressed. Essentially, $\lambda$ can be considered as a function of $x$. To see this, let $u(x)=1+x^{2}$. It follows from Lemma 2.6 that

$$
1+x^{2}=\Gamma(1-a) \lambda^{a}\left(\frac{\lambda^{-a}}{\Gamma(1-a)}+\frac{\lambda^{2-a}}{\Gamma(3-a)}\right)=1+\frac{2 \lambda^{2}}{(1-a)(2-a)} .
$$

From last equality, we get

$$
\lambda=\sqrt{\frac{(1-a)(2-a)}{2}} x \in(0, x)
$$

which shows that $\lambda$ is a function depending on $x$.

In the following, we present a Peano-type existence theorem for problem (1.1), We use Theorem 2.2 to prove the existence of the continuous solution of (1.1).

Theorem 2.8. Let (A.1) is satisfied and $r, T$ be fixed positive real numbers. Moreover, suppose that there exists a positive real number $M^{*}$ such that

$$
\left|x^{a} f(x, t)-\frac{u_{0}}{\Gamma(1-a)}\right| \leq M^{*} \max \left(x, \frac{\left|t-u_{0}\right|}{r}\right)
$$

holds for all $x \in[0, T]$ and for all $t \in\left[u_{0}-r, u_{0}+r\right]$. Then, (1.1) has at least one continuous solution on $\left[0, T_{0}\right]$, where

$$
T_{0}:=\left\{\begin{array}{cll}
\frac{r}{M^{*} \Gamma(1-a)} & , \text { if } & M^{*} \Gamma(1-a) \geq r \\
T & , \text { if } & M^{*} \Gamma(1-a) \leq r .
\end{array}\right.
$$

Proof. Let us first construct an appropriate closed convex ball of $C([0, T])$ to verify the conditions of Theorem 2.2. According to inequality (2.3) let us assume that

$$
\left|x^{a} f(x, t)-\frac{u_{0}}{\Gamma(1-a)}\right| \leq M^{*} x
$$

is fulfilled for all $x \in[0, T]$ and for all $t \in\left[u_{0}-r, u_{0}+r\right]$.

We define the ball

$$
B_{r}\left(u_{0}\right) \equiv\left\{u \in C\left[0, T_{0}\right]: \sup _{x \in\left[0, T_{0}\right]}\left|u(x)-u_{0}\right| \leq r\right\}
$$

where $M^{*} \Gamma(1-a) \geq r$. Then, for any $u \in B_{r}\left(u_{0}\right)$, from (2.4) one has

$$
\begin{aligned}
\left|\mathcal{M} u(x)-u_{0}\right| & \leq \frac{1}{\Gamma(a)} \int_{0}^{x} \frac{\left|f(\xi, u(\xi))-\xi^{-a} \frac{u_{0}}{\Gamma(1-a)}\right|}{(x-\xi)^{1-a}} d \xi \\
& =\frac{1}{\Gamma(a)} \int_{0}^{x} \frac{\left|\xi^{a} f(\xi, u(\xi))-\frac{u_{0}}{\Gamma(1-a)}\right|}{\xi^{a}(x-\xi)^{1-a}} d \xi \\
& \leq \frac{M^{*}}{\Gamma(a)} \int_{0}^{x} \frac{\xi}{\xi^{a}(x-\xi)^{1-a}} d \xi \\
& \leq M^{*}|x| \Gamma(2-a),
\end{aligned}
$$

estimating the right side of the above inequality by using the fact: $\Gamma(2-a)<\Gamma(1-a)$ for all $a \in(0,1)$, then we get

$$
\left|\mathcal{M} u(x)-u_{0}\right| \leq M^{*} T_{0} \Gamma(1-a) .
$$

It follows from the last inequality and by the definition of $T_{0}$, that

$$
\sup _{x \in\left[0, T_{0}\right]}\left|\mathcal{M} u(x)-u_{0}\right|<M^{*} \Gamma(1-a) T_{0} \leq r .
$$


On the other hand, if

$$
\left|x^{a} f(x, t)-\frac{u_{0}}{\Gamma(1-a)}\right| \leq \frac{M^{*}}{r}\left|t-u_{0}\right|
$$

holds for all $x \in[0, T]$ and for all $t \in\left[u_{0}-r, u_{0}+r\right]$, we construct $B_{r}$ such that

$$
B_{r}\left(u_{0}\right) \equiv\left\{u \in C[0, T]: \sup _{x \in[0, T]}\left|u(x)-u_{0}\right| \leq r\right\}
$$

for $M^{*} \Gamma(1-a) \leq r$. It follows from (2.6),

$$
\begin{aligned}
\left|\mathcal{M} u(x)-u_{0}\right| & \leq \frac{1}{\Gamma(a)} \int_{0}^{x} \frac{\left|\xi^{a} f(\xi, u(\xi))-\frac{u_{0}}{\Gamma(1-a)}\right|}{\xi^{a}(x-\xi)^{1-a}} d \xi \\
& \leq \frac{M^{*}}{r \Gamma(a)} \int_{0}^{x} \frac{\left|u(\xi)-u_{0}\right|}{\xi^{a}(x-\xi)^{1-a}} d \xi \\
& \leq \frac{M^{*}}{\Gamma(a)} \int_{0}^{x} \frac{1}{\xi^{a}(x-\xi)^{1-a}} d \xi .
\end{aligned}
$$

for any $u \in B_{r}$ and for all $x \in[0, T]$. So, we find that

$$
\sup _{x \in[0, T]}\left|\mathcal{M} u(x)-u_{0}\right| \leq M^{*} \Gamma(1-a) \leq r .
$$

From (2.5) and (2.7), we attain $\mathcal{M}\left(B_{r}\left(u_{0}\right)\right) \subset B_{r}\left(u_{0}\right)$ which completes the proof.

Theorem 2.9 (Existence and Uniqueness). Under the conditions of Theorem 2.5, suppose that the inequality

$$
x^{a}\left|f\left(x, t_{1}\right)-f\left(x, t_{2}\right)\right| \leq \frac{1}{\Gamma(1-a)}\left|t_{1}-t_{2}\right|
$$

holds for all $x \in[0, T], t_{1}, t_{2} \in \mathbb{R}$ and $0<a<1$. Then problem (1.1) admits a unique continuous solution on $[0, T]$.

Proof. We proved the existence of the solution in Theorem 2.5. Thus for the uniqueness, let (1.1) has two different continuous solutions $u_{1}$ and $u_{2}$. We initially assume $\omega(x) \not \equiv 0$, where

$$
\omega(x):=\left\{\begin{array}{cc}
\left|u_{1}(x)-u_{2}(x)\right|, & x>0 \\
0, & x=0
\end{array}\right.
$$

It is easily seen that $\omega(x)$ is nonnegative for all $x \in[0, T]$ and continuous for all these $x$ values except $x=0$. For the continuity of $\omega(x)$ at $x=0$, using variable substitution $\xi=x t$ and condition (A.1) respectively, we have

$$
\begin{aligned}
0 \leq \lim _{x \rightarrow 0^{+}} \omega(x) & =\lim _{x \rightarrow 0^{+}} \frac{1}{\Gamma(a)}\left|\int_{0}^{x} \frac{f\left(\xi, u_{1}(\xi)\right)-f\left(\xi, u_{2}(\xi)\right)}{(x-\xi)^{1-a}} d \xi\right| \\
& \leq \lim _{x \rightarrow 0^{+}} \frac{1}{\Gamma(a)}\left|\int_{0}^{1} \frac{(x t)^{a}\left[f\left(x t, u_{1}(x t)\right)-f\left(\xi, u_{2}(x t)\right)\right]}{t^{a}(1-t)^{1-a}} d t\right| \\
& \leq \frac{1}{\Gamma(a)} \int_{0}^{1} \frac{\lim _{x \rightarrow 0^{+}}\left|(x t)^{a}\left[f\left(x t, u_{1}(x t)\right)-f\left(\xi, u_{2}(x t)\right)\right]\right|}{t^{a}(1-t)^{1-a}} d t \\
& =0
\end{aligned}
$$

which simply means that $\lim _{x \rightarrow 0^{+}} \omega(x)=0=w(0)$. 
It is obvious that there exists a $\lambda \in(0, T]$ such that $\omega(\lambda) \neq 0$, i.e. $\omega(\lambda)>0$. By using Lemma 2.6 and inequality (2.8), we get

$$
\begin{aligned}
0<\omega(\lambda) & =\left|u_{1}(\lambda)-u_{2}(\lambda)\right| \\
& =\Gamma(1-a)\left|\lambda_{*}^{a} D^{a}\left(u_{1}-u_{2}\right)\left(\lambda_{*}\right)\right| \\
& =\Gamma(1-a)\left|f\left(\lambda_{*}, u_{1}\left(\lambda_{*}\right)\right)-f\left(\lambda_{*}, u_{2}\left(\lambda_{*}\right)\right)\right| \\
& \leq\left|u_{1}\left(\lambda_{*}\right)-u_{2}\left(\lambda_{*}\right)\right|=\omega\left(\lambda_{*}\right)
\end{aligned}
$$

for some $\lambda_{*} \in(0, \lambda)$. Applying the same procedure for the point $\lambda_{*}$ we find that, there exists some $\lambda_{2} \in\left(0, \lambda_{*}\right)$ such that $0<\omega(\lambda) \leq \omega\left(\lambda_{*}\right) \leq \omega\left(\lambda_{2}\right)$. Continuing in the same way, we construct a sequence $\left\{\lambda_{n}\right\}_{n=1}^{\infty} \subset[0, \lambda)$ with $\lambda_{1}=\lambda_{*}$ satisfying $\lambda_{n} \rightarrow 0$ and

$$
0<\omega(\lambda) \leq \omega\left(\lambda_{1}\right) \leq \omega\left(\lambda_{2}\right) \leq \ldots \leq \omega\left(\lambda_{n}\right) \leq \cdots
$$

On the other hand, since $\omega(x)$ is continuous at $x=0$ and $\lambda_{n} \rightarrow 0$, then $\omega\left(\lambda_{n}\right) \rightarrow \omega(0)=0$ that contradicts with (2.9). Hence $\omega(x) \equiv 0$, namely IVP (1.1) admits a unique continuous solution.

It is interesting to note that there are some other techniques and theorems (see, for example [7] and [22]) that enable us to replace a positive fixed real number larger than Nagumo constant or an arbitrary positive real number instead of Nagumo constant so that the corresponding IVPs admit a unique solution. However, the mentioned techniques and theorems could not be applied to problem (1.1), namely there does not exist a larger number than $\frac{1}{\Gamma(1-a)}$ in $(2.8)$ which can be replaced instead of $\frac{1}{\Gamma(1-a)}$ to guarantee the uniqueness of the continuous solution of (1.1). The following example may clearly express the foregoing discussion.

Example 2.10. Let us consider the function, $f_{\beta}(x, t):=\frac{\Gamma(\beta+1)}{\Gamma(1-a+\beta)} x^{-a}(t+k)$ where $k=$ $\frac{\Gamma(\beta-a+1)-\Gamma(1+\beta) \Gamma(1-a)}{\Gamma(1+\beta) \Gamma(1-a)}, \beta>0$ and $u_{0}=1$ in problem (1.1). It is clear that conditions (A.1) and (A.2) are satisfied. However, inequality (2.8) does not hold for $f_{\beta}(x, t)$, since $\frac{\Gamma(\beta+1)}{\Gamma(1-a+\beta)}$ replaces instead of $\frac{1}{\Gamma(1-a)}$ in $(2.8)$ and $\frac{\Gamma(\beta+1)}{\Gamma(1-a+\beta)}>\frac{1}{\Gamma(1-a)}$ for $\beta>0$ and $a \in(0,1)$. That is to say, the solution of (1.1) may not be unique. Indeed, (1.1) has infinitely many solutions $u(x)=c x^{\beta}+1$, where $c$ is an arbitrary real number. Furthermore, it is to be pointed out that $\frac{\Gamma(\beta+1)}{\Gamma(1-a+\beta)} \rightarrow \frac{1}{\Gamma(1-a)}$ and $f_{\beta}(x, t) \rightarrow f(x, t)=\frac{x^{-a} t}{\Gamma(1-a)}$ when $\beta \rightarrow 0$ and that, for $f(x, t)=\frac{x^{-a} t}{\Gamma(1-a)},(1.1)$ with $u_{0}=1$ admits a unique solution in the form $u(x)=1$.

\section{References}

[1] R.P. Agarwal and V. Lakshmikantham, Uniqueness and nonuniqueness criteria for ordinary differential equations, Series in Real Analysis, vol. 6, World Scientific, 1993.

[2] Z. Bai and H. Lü, Positive solutions for boundary value problem of nonlinear fractional differential equation, J. Math. Anal. Appl. 311 (2), 495-505, 2005.

[3] D. Baleanu and G.M. Octavian, On the existence interval for the initial value problem of a fractional differential equation, Hacet. J. Math. Stat. 40 (4), 2011.

[4] K. Deimling, Nonlinear functional analysis, Dover Publications, 464 pages, 2010.

[5] D. Delbosco and L. Rodino, Existence and uniqueness for a nonlinear fractional differential equation, J. Math. Anal. Appl. 204, 609-625, 1996.

[6] J.B. Diaz and W.L. Walter, On uniqueness theorems for ordinary differential equations and for partial differential equations of hyperbolic type, Trans. Amer. Math. Soc. 96, 90-100, 1960.

[7] K. Diethelm, The mean value theorems and a Nagumo-type uniqueness theorem for Caputo's fractional calculus, Fract. Calc. Appl. Anal. 15 (2), 304-313, 2012. 
[8] P. Drábek and A. Fonda, Handbook of differential equations: ordinary differential equations, vol 3, Elsevier, North Holland, 2006.

[9] J. Dugundji and A. Granas, Fixed point theory Mathematical Monographs, 61, Państwowe Wydawnictwo Naukowe, PWN, Warszawa, 1982.

[10] P.W. Eloe and T. Masthay, Initial value problems for Caputo fractional differential equations, J. Fract. Calc. Appl. 9 (2), 178-195, 2018.

[11] R.A.C. Ferreira, A Nagumo-type uniqueness result for an nth order differential equation, Bull. Lond. Math. Soc. 45 (5), 930-934, 2013.

[12] A.F. Filippov, Differential equations with discontinuous right-hand sides: control systems, vol. 18, Springer Science \& Business Media, 2013.

[13] N. Heymans and I. Podlubny, Physical interpretation of initial conditions for fractional differential equations with Riemann-Liouville fractional derivatives, Rheologica Acta. 45 (5), 765-771, 2006.

[14] A.A. Kilbas, H.M. Srivasta and J.J. Trujilllo, Theory and applications of fractional differential equations, North-Holland Mathematics Studies, vol. 204, Elsevier Science Limited, 2006.

[15] V. Lakshmikantham and S. Leela, Nagumo-type uniqueness result for fractional differential equations, Nonlinear Anal. 71 (7-8), 2886-2889, 2009.

[16] V. Lakshmikanthan and A.S. Vatsala, Basic theory of fractional differential equations, Nonlinear Anal. 69, 2677-2682, 2008.

[17] Z.M. Odibat and N.T. Shawagfeh, Generalized Taylor's formula, Appl. Math. Comput. 186 (1), 286-293, 2007.

[18] M.D. Ortigueira, Fractional calculus for scientists and engineers, vol. 84, Springer, Dordrecht, 2011.

[19] M.D. Ortigueira and F.J. Coito, System initial conditions vs derivative initial conditions, Comput. Math. Appl. 59 (5), 1782-1789, 2010.

[20] I. Podlubny, Fractional differential equations: an introduction to fractional derivatives, fractional differential equations, to methods of their solution and some of their applications, 198, Elsevier, 1998.

[21] S.G. Samko, A.A. Kilbas and O.I. Marichev, Fractional Integrals and Derivatives: Theory and Applications, Gordon and Breach Science Publishers, Yverdon, Switzerland, 1993.

[22] C. Sin and Z. Liancun, Existence and uniqueness of global solutions of Caputo-type fractional differential equations, Fract. Calc. Appl. Anal. 19 (3), 765-774, 2016.

[23] M. Şan, Complex variable approach to the analysis of a fractional differential equation in the real line, C. R. Math. Acad. Sci. Paris, 356 (3), 293-300, 2018.

[24] M. Şan and K.N. Soltanov, The New Existence and Uniqueness Results for Complex Nonlinear Fractional Differential Equation, arXiv preprint arXiv:1512.04780.

[25] T. Trif, Existence of solutions to initial value problems for nonlinear fractional differential equations on the semi-axis, Fract. Calc. Appl. Anal. 16 (3), 595-612, 2013.

[26] S. Zhang, Monotone iterative method for initial value problem involving RiemannLiouville fractional derivatives, Nonlinear Anal. 71, 2087-2093, 2009. 